\section{Prevalência de hipertensão arterial sistêmica e fatores associados: um estudo de base populacional em mulheres no Sul do Brasil}

\author{
Prevalence of systemic hypertension and \\ associated factors: a population-based \\ study among women in the \\ South of Brazil
}

\author{
Milton Hartmann 1 \\ Juvenal Soares Dias-da-Costa 1,2 \\ Maria Teresa Anselmo Olinto 1 \\ Marcos Pascoal Pattussi 1 \\ Ângela Tramontini 1
}

\author{
1 Programa de Pós-graduação \\ em Saúde Coletiva, \\ Universidade do Vale do \\ Rio dos Sinos, \\ São Leopoldo, Brasil. \\ 2 Faculdade de Medicina, \\ Universidade Federal de \\ Pelotas, Pelotas, Brasil. \\ Correspondência \\ J. S. Dias-da-Costa \\ Faculdade de Medicina, \\ Universidade Federal de \\ Pelotas. \\ Av. Duque de Caxias 250, \\ Pelotas, RS \\ 96030 002, Brasil. \\ jcosta@epidemio-ufpel.org.br
}

\begin{abstract}
A cross-sectional study was carried out among women 20 to 60 years of age living in São Leopoldo, Rio Grande do Sul State, Brazil, to determine the prevalence of hypertension and associated factors. Census tract sampling was used, and the outcome was defined by the presence of arterial pressure $\geq 140 / 90 \mathrm{mmHg}$ and/or use of antihypertensive drugs. Among 1,020 interviewed people, 267 (26.2\%; 95\%CI: 23.5-28.9) presented hypertension. Poisson regression showed a statistically significant association with age, skin color, marital status, family history, educational background, and nutritional status. The highest attributable fractions were observed among women with schooling $\leq 4$ years (18.38\%); obese women (33.86\%); and overweight women (22.55\%). The results were compared to other hypertension prevalence studies in the State of Rio Grande do Sul, and the results showed the need for inter-sector efforts, highlighting the possible contribution of increasing people's level of schooling. The study also showed the relevance of weight loss measures in the adult population.
\end{abstract}

Hypertension; Cross-Sectional Studies; Women; Risk Factors

\section{Introdução}

As doenças cardiovasculares são as principais causas de morte em todo o mundo 1 . No Brasil, as doenças do aparelho circulatório são também as principais causas de óbito para todas as idades em ambos os sexos 2 , fato que se repete no Estado do Rio Grande do Sul, sendo que, durante o ano de 2002, a mortalidade proporcional foi de $31,6 \%{ }^{3}$. Em São Leopoldo, foi observada a mesma tendência, durante o ano 2000, a principal causa de mortalidade para todas as idades e em ambos os sexos foram as doenças do aparelho circulatório ${ }^{4}$. Sabe-se que a hipertensão arterial pode ser considerada causa direta ou fator de risco para outras doenças do coração, principalmente cardiopatia isquêmica e doença cerebrovascular. No Município de São Paulo, um estudo realizado por Chiavegatto Filho et al. 5 , em mulheres de 20 a 49 anos, mostrou que hipertensão era causa associada em 53,1\% dos óbitos.

Segundo Lessa 6, ocorreram no Brasil, de janeiro de 1998 a junho de 2001, 204.227 internações hospitalares de mulheres por hipertensão e 339.825 de homens, atingindo respectivamente, $11,6 \%$ e $17,4 \%$ das internações por doenças cardiovasculares específicas.

Assim, a prevalência na população, a gravidade das conseqüências e seu elevado custo para o sistema de saúde, justificam os estudos sobre hipertensão arterial e seus fatores de risco. 
O presente estudo tem como objetivos estimar a prevalência de hipertensão arterial na população de mulheres de 20 a 60 anos da cidade de São Leopoldo, no Estado do Rio Grande do Sul, e determinar alguns fatores associados.

\section{Material e métodos}

Este estudo transversal faz parte do projeto de pesquisa Condições de Saúde das Mulheres: Estudo de Base Populacional na Região do Vale do Rio dos Sinos, realizado pela Universidade do Vale do Rio dos Sinos através do Programa de Pósgraduação em Saúde Coletiva, no ano de 2003. Neste trabalho, buscou-se caracterizar a saúde das mulheres residentes na zona urbana de São Leopoldo, na faixa etária de 20 a 60 anos. O projeto de pesquisa foi aprovado pelo Comitê de Ética e Pesquisa da Faculdade de Medicina da Universidade Federal de Pelotas.

A cidade de São Leopoldo situa-se na Região Metropolitana de Porto Alegre, no Vale do Rio dos Sinos, no Estado do Rio Grande do Sul. O município tinha, segundo o último censo demográfico, 193.547 habitantes, sendo 98.781 mulheres (51,07\%).

Estimou-se a amostra a partir de diferentes desfechos previstos neste projeto, escolhendose aquele com maior número de mulheres (prevalência de diabetes mellitus). Calculou-se um tamanho de amostra que permitisse identificar uma razão de risco de 2,0, para um nível de confiança de $95 \%$, de um poder de $80 \%$, mantida a razão de não-expostos: expostos de 1:3.

Optou-se pelo maior tamanho de amostra: 1.086 pessoas. Para compensar possíveis perdas, aumentou-se a amostra em $10 \%$ e adicionou-se $15 \%$ para controlar fatores de confusão, totalizando 1.358 pessoas. Conhecendo-se o número de pessoas necessário para a amostra, a média de pessoas por domicílio na cidade de São Leopoldo $(3,56)$ e a proporção de mulheres na faixa etária de interesse (30\%), foram visitadas 1.440 residências 7 .

Ao final do trabalho de campo foram incluídas 1.026 mulheres no estudo, sendo que 58 $(5,6 \%)$ foram classificadas como perdas ou recusas. Entre o total de mulheres participantes, seis não tiveram medidas de pressão arterial aferidas, sendo excluídas da análise.

Às mulheres incluídas no estudo foram aplicados questionários padronizados, pré-codificados e pré-testados. Os entrevistadores receberam treinamento para aplicação dos questionários e coleta das medidas de peso, altura e pressão arterial. A coleta de dados foi realizada de março a novembro de 2003.
O controle de qualidade da coleta dos dados foi realizado em $10 \%$ da amostra, utilizando-se questionário simplificado, composto por variáveis que não se alteravam em curto espaço de tempo.

A pressão arterial de cada mulher incluída no estudo foi medida duas vezes durante a aplicação do questionário. As medidas das pressões sistólicas e diastólicas corresponderam às suas médias aritméticas, e foram efetuadas no meio e no final do questionário. Utilizou-se estetoscópio e esfigmomanômetro aneróide para se efetuar a medida no braço direito, estando a entrevistada sentada, sem fumar, consumir café, chimarrão ou outro alimento qualquer durante a entrevista. Os entrevistadores foram treinados na aferição da pressão arterial, de acordo com o III Consenso Brasileiro de Hipertensão Arterial 8.

Foi investigado também o uso de medicamentos anti-hipertensivos.

Dessa forma, a variável de interesse, presença de hipertensão arterial, foi classificada de acordo com os níveis tensionais da Joint National Committee (JNC) 9 e/ou com o uso de medicação anti-hipertensiva. Portanto, as mulheres com níveis tensionais iguais ou maiores que 140x90mmHg e/ou com medidas tensionais abaixo destes níveis mas com uso de medicação anti-hipertensiva foram classificadas como hipertensas.

Foram exploradas as associações entre hipertensão arterial com variáveis demográficas, sócio-econômicas, obstétricas, morbidades e hábitos de vida. As variáveis demográficas incluídas na análise foram idade, cor da pele observada pelo entrevistador, estado civil e história familiar de hipertensão. Em relação à cor da pele, apenas 47 mulheres foram classificadas como negras $(4,6 \%)$, assim na análise foram consideradas duas categorias: brancas e não-brancas.

As variáveis sócio-econômicas analisadas foram: classificação econômica, escolaridade e renda familiar per capita. As classes econômicas foram definidas com base na tabela de classificação econômica da Associação Nacional de Empresas de Pesquisas 10, estabelecida no Critério de Classificação Econômica Brasil, a partir da posse de bens materiais, da escolaridade do chefe da família e da presença de empregados domésticos nas residências.

Foi analisado o uso ou não de anticoncepcionais orais no momento da entrevista, sem discriminar tipo, quantidade e tempo de uso.

Entre os hábitos de vida e as morbidades foram analisadas as seguintes variáveis: tabagismo, estado nutricional, uso de sal adicional nas refeições, atividade física, abuso de álcool e presença de diabetes. 
Considerou-se como fumante o indivíduo que faz uso de cigarro diariamente, independente da quantidade.

Verificou-se o estado nutricional usando-se o cálculo do índice de massa corporal (IMC = peso em kg/altura ${ }^{2} \mathrm{em} \mathrm{m}$ ), sendo consideradas com sobrepeso as mulheres com IMC entre 25,0 e 29,9kg/ $\mathrm{m}^{2}$, e as obesas com $30,0 \mathrm{~kg} / \mathrm{m}^{2}$ ou mais 11 .

Foram classificadas como sedentárias as mulheres que praticavam atividade física uma vez por semana ou tinham atividades diárias consideradas de baixo consumo energético, e não-sedentárias ou ativas as que praticavam duas ou mais vezes por semana, no mínimo dez minutos de duração 12 .

A variável consumo de álcool foi criada com base na freqüência, tipo de bebida e quantidade ingerida. O consumo abusivo de álcool foi definido a partir da ingestão de 30g/dia de etanol ou mais 13 .

A entrada dos dados foi realizada usando-se o programa Epi Info (Centers for Disease Control and Prevention, Atlanta, Estados Unidos), em dupla entrada e posterior comparação, para se eliminar a probabilidade de erros de digitação. A análise univariada dos dados foi realizada utilizando-se o programa SPSS, versão 11.0 (SPSS Inc., Chicago, Estados Unidos). O efeito de delineamento e a correção das medidas de efeito, com os respectivos intervalos de confiança e testes de associação foram calculados no programa Stata, versão 7.0 (Stata Corp., College Station, Estados Unidos). Foram selecionadas variáveis para análise multivariada, através de regressão de Poisson (p-valor $<0,2$ ), conforme modelo hierarquizado (Figura 1 ).

Foram calculadas as frações atribuíveis para os fatores associados modificáveis entre a população, ou seja, a proporção de prevalência de hipertensão arterial que poderia ser reduzida se a exposição fosse eliminada. A fração atribuível foi estimada pela fórmula $\mathrm{P}_{\mathrm{e}}(\mathrm{RP}-1) / 1+\mathrm{P}_{\mathrm{e}}$ (RP - 1), onde $\mathrm{P}_{\mathrm{e}}$ é a proporção de exposição na população, RP é prevalência da doença nos expostos/prevalência da doença entre os não-expostos 14 .

\section{Resultados}

Entre as 1.020 pessoas entrevistadas, 267 (26,2\%; IC95\%: 23,5-28,9) apresentavam hipertensão arterial sistêmica segundo os critérios do estudo.

Em relação às variáveis demográficas constatou-se que mais de $50 \%$ das mulheres tinham idade situada entre 20 e 39 anos, a grande maioria das entrevistadas era de cor branca (83\%) e $64 \%$ era casada ou referiu união estável. Quanto à história familiar de hipertensão, mais da metade das mulheres (51\%) respondeu ter pelo menos um dos pais hipertensos (Tabela 1).

Quanto às variáveis sócio-econômicas, $44 \%$ das mulheres possuíam até sete anos de estudo e $14 \%$ apresentaram 14 anos ou mais. Os dados mostraram que as pessoas classificadas nas classes C, D e E constituíram 2/3 da amostra (66,6\%) e a grande maioria (77\%) tinha renda familiar igual ou menor que três salários mínimos (Tabela 1).

Para analisar a variável uso de anticoncepcional oral foram incluídas apenas as mulheres em idade reprodutiva, sendo que a maioria não fazia uso (54\%) (Tabela 1).

Em relação às morbidades e hábitos de vida, verificou-se que mais de 3/4 das entrevistadas eram de não-fumantes ou de ex-fumantes (77\%), metade das mulheres apresentava estado nutricional normal ou baixopeso (50\%) e em sua maioria (68\%) não praticava atividade física. A maioria das mulheres respondeu não fazer uso de álcool (93\%), não apresentava diabetes (96\%) e não adicionava sal à comida depois de pronta (93\%) (Tabela 1).

A amostragem foi por conglomerados exigindo o cálculo do efeito de delineamento da variável desfecho (tensão arterial maior ou igual a 140x90mmHg e/ou uso de medicação anti-hipertensiva) usando-se o programa Stata. O efeito do delineamento para o desfecho nos setores sorteados foi de 2,07, sendo considerado para o cálculo das medidas de efeito nas análises.

$\mathrm{Na}$ análise bivariada foram observadas associações com idade, estado civil e história familiar de hipertensão arterial. À medida que aumentava a idade, aumentava a prevalência de hipertensão arterial, com teste de tendência linear significativo. Constatou-se que as mulheres solteiras apresentavam menor prevalência de hipertensão arterial. Quando relacionada à história familiar de hipertensão, verificou-se que nas entrevistadas com um dos pais hipertensos a prevalência era quase $50 \%$ maior $(26,7 \%)$, e as mulheres com ambos os pais hipertensos apresentavam mais que o dobro $(40,6 \%)$ do que as que não tinham história familiar (18\%) (Tabela 1).

Em relação às variáveis sócio-econômicas, observou-se associação entre hipertensão e escolaridade. As prevalências de hipertensão foram aumentando na medida em que os anos de estudo diminuíam. Observou-se que as mulheres com sete anos ou menos de estudo apresentaram intervalos de confiança acima do valor unitário. Deve-se ressaltar que a associação entre escolaridade e hipertensão apresentou teste de tendência linear significativo; a cada diminuição no nível de escolaridade correspondia a um aumento na prevalência de hipertensão 
Modelo hierarquizado de análise.

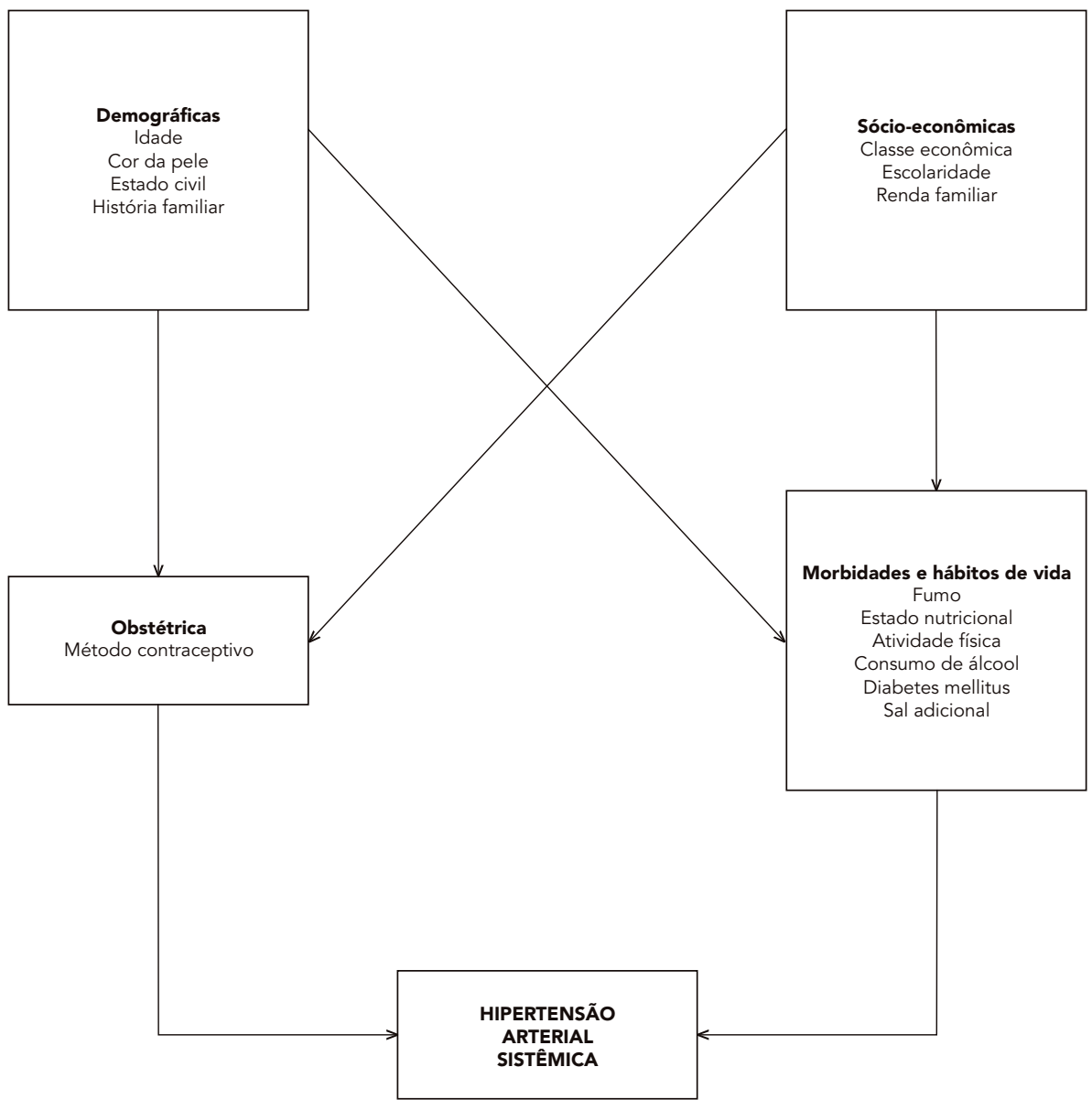

arterial, equivalente a um gradiente de dose resposta (Tabela 1).

Embora as outras variáveis sócio-econômicas não tenham revelado associação com hipertensão arterial, foi verificado teste de tendência linear significativo para classe econômica, mostrando a cada mudança de categoria um aumento da prevalência de hipertensão (Tabela 1).

As mulheres que faziam uso de anticoncepcional oral apresentaram prevalência de hipertensão menor (Tabela 1).

O estudo revelou associação da hipertensão com estado nutricional das mulheres. As mulheres com sobrepeso tiveram uma prevalência quase duas vezes maior e as obesas quase quatro vezes mais do que as classificadas com peso normal/baixo. Deve-se destacar que a prevalência de hipertensão entre as mulheres com obesidade foi de $56 \%$, ou seja, a maior medida de freqüência observada no estudo. Constatou-se, também, a associação de hipertensão entre as mulheres que referiram presença de diabetes mellitus, a razão de prevalência nesta categoria foi o dobro da categoria de base (Tabela 1).

As variáveis cor da pele, classe econômica, renda familiar, fumo, atividade física, consumo de álcool e consumo de sal não mostraram associação estatística com a hipertensão arterial. Entretanto, deve-se destacar que as variáveis: cor da pele, classe econômica, consumo de álcool e uso de sal adicional apresentaram p-valor $<0,20$ e, portanto, ingressaram no modelo de análise multivariada. 
Distribuição de hipertensão em relação às variáveis demográficas, sócio-econômicas, obstétricas, morbidades e hábitos de vida. São Leopoldo, Rio Grande do Sul, Brasil, 2003.

\begin{tabular}{|c|c|c|c|c|c|}
\hline Variável & $\mathrm{N}(\%)$ & Prevalência de HAS (\%) & RP & IC95\% & p-valor \\
\hline Idade $($ anos)* & & & & & $<0,0001$ \\
\hline $20-29$ & $283(27,7)$ & $24(8,5)$ & 1 & & \\
\hline $30-39$ & $253(24,8)$ & $42(16,6)$ & 1,96 & $1,16-3,30$ & \\
\hline $40-49$ & $300(29,4)$ & $101(33,7)$ & 3,97 & $2,74-5,75$ & \\
\hline $50-60$ & $184(18,0)$ & $100(54,3)$ & 6,41 & $4,27-9,61$ & \\
\hline Cor da pele & & & & & 0,21 \\
\hline Branca & $855(83,8)$ & $218(25,5)$ & 1 & & \\
\hline Não-branca & $165(16,2)$ & $49(29,7)$ & 1,16 & $0,91-1,48$ & \\
\hline Estado civil & & & & & $<0,0001$ \\
\hline Casada/Em união & $656(64,3)$ & $197(30,0)$ & 1 & & \\
\hline Viúva/Separada/Divorciada & $145(14,2)$ & $47(32,4)$ & 1,08 & $0,84-1,39$ & \\
\hline Solteira & $219(21,5)$ & $23(10,5)$ & 0,35 & $0,24-0,50$ & \\
\hline História familiar de hipertensão & & & & & 0,0001 \\
\hline Não & $322(33,4)$ & $58(18,0)$ & 1 & & \\
\hline Pai ou mãe & $499(51,8)$ & $133(26,7)$ & 1,48 & $1,10-1,98$ & \\
\hline Ambos & $143(14,8)$ & $58(40,6)$ & 2,25 & $1,58-3,20$ & \\
\hline Escolaridade (anos) * & & & & & $<0,0001$ \\
\hline $14-23$ & $145(14,2)$ & $27(18,6)$ & 1 & & \\
\hline $11-13$ & $257(25,2)$ & $38(14,8)$ & 0,79 & $0,48-1,30$ & \\
\hline $8-10$ & $169(16,6)$ & $43(25,4)$ & 1,37 & $0,87-2,14$ & \\
\hline $5-7$ & $244(23,9)$ & $78(32,0)$ & 1,71 & $1,10-2,68$ & \\
\hline $0-4$ & $205(20,1)$ & $81(39,5)$ & 2,12 & $1,41-3,19$ & \\
\hline Classe econômica ANEP ** & & & & & 0,07 \\
\hline Classe A & $74(7,3)$ & $18(24,3)$ & 1 & & \\
\hline Classe B & $265(26,1)$ & $60(22,6)$ & 0,93 & $0,60-1,44$ & \\
\hline Classe C & $401(39,4)$ & $101(25,2)$ & 1,03 & $0,68-1,58$ & \\
\hline Classes D e E & $277(27,2)$ & $88(31,8)$ & 1,31 & $0,85-2,01$ & \\
\hline Renda familiar (salários mínimos) & & & & & 0,39 \\
\hline 6,01 ou + & $85(8,3)$ & $15(17,6)$ & 1 & & \\
\hline $3,01-6,00$ & $141(13,8)$ & $36(25,5)$ & 1,45 & $0,83-2,51$ & \\
\hline $1,01-3,00$ & $425(41,7)$ & $112(26,4)$ & 1,49 & $0,87-2,57$ & \\
\hline $0,00-1,00$ & $369(36,2)$ & $104(28,2)$ & 1,6 & $0,94-2,72$ & \\
\hline Anticoncepcional oral & & & & & 0,01 \\
\hline Não & $341(54,7)$ & $90(26,4)$ & 1 & & \\
\hline Sim & $282(45,3)$ & $51(18,1)$ & 0,68 & $0,52-0,90$ & \\
\hline Fumo & & & & & 0,71 \\
\hline Nunca fumou & $603(59,1)$ & $153(25,4)$ & 1 & & \\
\hline Ex-fumante & $187(18,3)$ & $53(28,3)$ & 1,12 & $0,85-1,46$ & \\
\hline Fumante & $230(22,5)$ & $61(26,5)$ & 1,04 & $0,81-1,35$ & \\
\hline Estado nutricional & & & & & $<0,0001$ \\
\hline Normal/Baixo peso & $504(50,8)$ & $74(14,7)$ & 1 & & \\
\hline Sobrepeso & $311(31,3)$ & $88(28,3)$ & 1,93 & $1,51-2,45$ & \\
\hline Obesidade & $178(17,9)$ & $101(56,7)$ & 3,86 & $3,10-4,81$ & \\
\hline Atividade física & & & & & 0,63 \\
\hline Sim & $323(31,7)$ & $81(25,1)$ & 1 & & \\
\hline Não & $697(68,3)$ & $186(26,7)$ & 1,06 & $0,82-1,34$ & \\
\hline
\end{tabular}

(continua) 


\begin{tabular}{|c|c|c|c|c|c|}
\hline Variável & $\mathrm{N}(\%)$ & Prevalência de HAS (\%) & $\mathrm{RP}$ & IC95\% & p-valor \\
\hline Consumo de álcool (g/dia) & & & & & 0,21 \\
\hline$<30$ & $954(93,5)$ & $255(26,7)$ & 1 & & \\
\hline$\geq 30$ & $66(6,5)$ & $12(18,2)$ & 0,69 & $0,38-1,25$ & \\
\hline Diabete mellitus & & & & & $<0,0001$ \\
\hline Não & $982(96,3)$ & $247(25,2)$ & 1 & & \\
\hline Sim & $38(3,7)$ & $20(52,6)$ & 2,09 & $1,54-2,84$ & \\
\hline Sal adicional & & & & & 0,13 \\
\hline Não & $954(93,5)$ & $255(26,7)$ & 1 & & \\
\hline Sim & $66(6,5)$ & $12(18,2)$ & 0,68 & $0,41-1,13$ & \\
\hline
\end{tabular}

HAS: hipertensão arterial sistêmica; RP: razão de prevalência; IC95\%: intervalo de confiança de 95\%; ANEP: Associação Nacional de Empresas de Pesquisa.

* Teste de tendência linear $<0,001$.

** Teste de tendência linear $<0,05$.

A análise multivariada realizada por meio da regressão de Poisson, seguindo o modelo hierarquizado mostrou associação estatisticamente significativa para as variáveis idade, cor da pele, estado civil, história familiar de hipertensão, escolaridade e estado nutricional. As medidas de efeito mais elevadas foram observadas na associação de idade, principalmente, a partir dos 40 anos e nas mulheres classificadas como nãobrancas (Tabela 2).

A partir das razões de prevalência obtidas na análise multivariada foram calculadas as frações atribuíveis na população para as categorias das variáveis escolaridade e obesidade. As maiores frações atribuíveis foram observadas entre as mulheres com até quatro anos de escolaridade $(18,38 \%)$; entre as obesas $(33,86 \%)$ e mulheres com sobrepeso $(22,55 \%)$ (Tabela 3$)$.

\section{Discussão}

Diversos estudos de prevalência foram conduzidos em todo o Brasil e no Estado do Rio Grande do Sul. Contudo, a presente investigação introduziu nas apresentações das razões de prevalência o efeito de delineamento, e a análise multivariada foi realizada por meio da regressão de Poisson demonstrando a magnitude das associações sem problemas de estimativa, oriundas da utilização de razões de odds 15 .

Segundo Lessa 16 (p. 81), "a magnitude da hipertensão varia amplamente a depender dos atributos biológico-demográficos das populações, do estilo de vida predominante em cada uma delas, do ambiente físico e psicossocial e até das características da organização dos serviços de saúde e das respectivas interações entre esses vários elementos". Assim, a revisão bibliográfica deste projeto foi fundamentalmente focada nos estudos de prevalência de hipertensão arterial realizados no Estado do Rio Grande do Sul 17,18,19,20,21,22.

As medidas de desfecho nos estudos de prevalência, realizados no Rio Grande do Sul, apresentaram variações. Achutti et al. 17, Duncan et al. 18, Piccini \& Victora 21, Trindade et al. 22, por exemplo, utilizaram como pontos de corte as medidas $\geq 160 \mathrm{mmHg}$ para pressão sistólica e $\geq 95 \mathrm{mmHg}$ para pressão diastólica. Mais recentemente, Fuchs et al. 19 e Gus et al. 20 utilizaram medidas de pressão $\geq 140$ e $\geq 90$ para determinação de pressão arterial. Assim, a prevalência de hipertensão arterial encontrada no presente estudo foi superior às prevalências encontradas por Achutti et al. 17 (11\%; IC95\%: 10,26-12,24) e Duncan et al. 18 (15\%; IC95\%: 12-18). Contudo, foi inferior à prevalência total para o Estado do Rio Grande do Sul estabelecida no estudo de Gus et al. 20 (33,7\%; IC95\%: 30,6-36,8). A comparação com os demais estudos realizados no Rio Grande do Sul não evidenciou diferenças quanto à prevalência de hipertensão arterial.

Assim como nos outros estudos realizados no Rio Grande do Sul, a análise revelou associação entre hipertensão e idade. Na regressão de Poisson a prevalência de hipertensão arterial nas mulheres maiores de 50 anos era cinco vezes maior do que nas mais jovens. Vale destacar que os intervalos de confiança dos porcentuais das distribuições por idade no presente estudo coincidem com a distribuição da população do Município de São Leopoldo verificada no recenseamento de 2000, conferindo validade externa aos resultados.

Em todos os estudos a variável cor da pele foi dicotomizada em duas categorias, sendo definidas a partir da observação dos entrevistadores. Como no presente estudo, foram encontradas as- 
Análise multivariada através de regressão de Poisson para hipertensão e variáveis demográficas, sócio-econômicas, obstétricas, morbidades e hábitos de vida. São Leopoldo, Rio Grande do Sul, Brasil, 2003.

\begin{tabular}{|c|c|c|c|}
\hline Variável & RP & IC95\% & p-valor \\
\hline \multicolumn{4}{|l|}{ Idade (anos) } \\
\hline $20-29$ & 1 & & \\
\hline $30-39$ & 1,53 & $0,91-2,57$ & 0,103 \\
\hline $40-49$ & 3,28 & $2,25-4,75$ & $<0,0001$ \\
\hline $50-60$ & 5,22 & $3,49-7,83$ & $<0,0001$ \\
\hline \multicolumn{4}{|l|}{ Cor da pele } \\
\hline Branca & 1 & & \\
\hline Não-branca & 2,74 & $1,08-1,67$ & 0,009 \\
\hline \multicolumn{4}{|l|}{ Estado civil } \\
\hline Casada/Em união & 1 & & \\
\hline Viúva/Separada/Divorciada & 0,82 & $0,66-1,03$ & 0,084 \\
\hline Solteira & 0,56 & $0,38-0,82$ & 0,004 \\
\hline \multicolumn{4}{|l|}{ História familiar de hipertensão } \\
\hline Não & 1 & & \\
\hline Pai ou mãe & 1,32 & $0,99-1,75$ & 0,054 \\
\hline Ambos & 1,85 & $1,34-2,56$ & $<0,0001$ \\
\hline \multicolumn{4}{|l|}{ Escolaridade (anos) } \\
\hline $14-23$ & 1 & & \\
\hline $11-13$ & 0,91 & $0,55-1,50$ & 0,722 \\
\hline $8-10$ & 1,65 & $1,03-2,63$ & 0,037 \\
\hline $5-7$ & 2,1 & $1,31-3,38$ & 0,003 \\
\hline $0-4$ & 2,66 & $1,69-4,18$ & $<0,0001$ \\
\hline \multicolumn{4}{|l|}{ Anticoncepcional oral } \\
\hline Não & 1 & & \\
\hline Sim & 1,14 & $0,83-1,58$ & 0,41 \\
\hline \multicolumn{4}{|l|}{ Estado nutricional * } \\
\hline Normal/Baixo peso & 1 & & \\
\hline Sobrepeso & 1,56 & $1,19-2,04$ & 0,002 \\
\hline Obesidade & 2,47 & $1,87-3,26$ & $<0,0001$ \\
\hline \multicolumn{4}{|l|}{ Consumo de álcool (g/dia) } \\
\hline$<30$ & 1 & & \\
\hline$\geq 30$ & 1 & $0,60-1,68$ & 0,99 \\
\hline \multicolumn{4}{|l|}{ Diabete mellitus } \\
\hline Não & 1 & & \\
\hline Sim & 1,14 & $0,84-1,53$ & 0,38 \\
\hline \multicolumn{4}{|l|}{ Sal adicional } \\
\hline Não & 1 & & \\
\hline $\operatorname{Sim}$ & 1,09 & $0,64-1,84$ & 0,75 \\
\hline
\end{tabular}

* Efeito ajustado para idade, cor da pele, estado civil, história familiar de hipertensão, escolaridade.

sociações nas investigações de Achutti et al. 17 e Piccini \& Victora 21 mostrando prevalências mais elevadas entre os não-brancos.

Incluiu-se a variável estado civil na análise, pois a presença de companheiro ou de laços familiares estáveis estava relacionada com acesso aos serviços de saúde 23,24, capacidade de evitar hospitalizações por hipertensão 25 e poderia estar associada à presença da doença. Entretanto, mesmo após o controle para variáveis como idade, cor da pele e escolaridade, as mulheres solteiras apresentaram menor prevalência de hipertensão.

A associação com história familiar de hipertensão foi compatível com os estudos de Fuchs et al. 19 e Piccini \& Victora 21. 
Tabela 3

Frações atribuíveis para fatores associados modificáveis entre os expostos. São Leopoldo, Rio Grande do Sul, Brasil, 2003.

\begin{tabular}{lc}
\hline Variável & Fração atribuível (\%) \\
\hline Escolaridade (anos) & \\
$8-10$ & 5,79 \\
$5-7$ & 14,51 \\
$0-4$ & 18,38 \\
Estado nutricional & \\
Sobrepeso & 22,55 \\
Obesidade & 33,86 \\
\hline
\end{tabular}

Neste estudo, os dois únicos fatores associados com hipertensão que poderiam ser classificados como modificáveis foram escolaridade $\mathrm{e}$ estado nutricional. A associação entre baixa escolaridade e hipertensão, encontrada também por Fuchs et al. 19, Gus et al. 20 e Piccini \& Victora 21 , pode expressar que más condições de vida predispõem o aparecimento de doenças. Devese lembrar que talvez o presente estudo não tenha tido o poder de detectar associação com as outras variáveis sócio-econômicas, que a análise evidenciou a presença de tendência linear.

A relação entre sobrepeso e obesidade com hipertensão arterial confirma os achados já revelados por Fuchs et al. 19, Gus et al. 20, Piccini \& Victora 21 e Trindade et al. 22. Essa associação contribui para adicionar evidências quanto às conseqüências do excesso de peso para a saúde dos adultos 26,27 .

A utilização das razões de prevalências dos fatores de risco modificáveis na análise multivariada permitiu o cálculo das frações atribuíveis entre as categorias que podem representar importante contribuição para a projeção de progra- mas de saúde pública 28 , na medida em que estimam os porcentuais de doença que poderiam ser reduzidos caso a exposição fosse alterada. Assim, os resultados mostraram a importância da necessidade de articulação inter-setorial, revelando a contribuição possível de ser obtida mediante elevação dos anos de escolaridade, bem como salientaram a relevância de se providenciar medidas de diminuição de peso na população adulta. Contudo, deve-se ressaltar que outros fatores envolvidos na etiologia da hipertensão arterial sistêmica, como hábitos alimentares e estresse, não foram incluídos no modelo de análise e que a diminuição de peso na população adulta depende de questões sociais, culturais e ambientais, que determinam os estilos de vida e que não dependem exclusivamente da atuação de profissionais de saúde.

O presente estudo, como os demais realizados no Estado do Rio Grande do Sul, investigou e não revelou associação com outros fatores relacionados com as doenças cardiovasculares, tais como presença de diabetes mellitus, sedentarismo, uso de sal adicional, consumo abusivo de álcool, uso de anticoncepcional oral e fumo. No presente estudo as variáveis sobre fumo e sedentarismo, por exemplo, apresentaram problemas na coleta. O questionário era um instrumento que precisava atender a inúmeros desfechos, assim, por motivos logísticos a coleta sobre tabaco não incluiu o tipo de tabaco consumido, o número de cigarros ou tempo de exposição ao hábito. De forma semelhante não foi investigado o tempo total gasto em atividades físicas no lazer, considerando-se como ativas as mulheres que poderiam desenvolver tais práticas duas vezes por semana durante dez minutos. Espera-se que com o transcorrer do tempo a coleta dessas variáveis se aperfeiçoe, além disso, os estudos de corte transversal apresentam problemas de direcionalidade entre exposições e desfechos e de causalidade reversa que podem mascarar tais efeitos. 


\section{Resumo}

Foi realizado um estudo transversal entre a população de mulheres de 20 a 60 anos residente na cidade de São Leopoldo, Rio Grande do Sul, Brasil, com o objetivo de determinar a prevalência de hipertensão arterial e fatores associados. A amostragem foi por conglomerados, e o desfecho foi definido pela presença de níveis tensionais iguais ou maiores que $140 \times 90 \mathrm{mmHg}$ e/ou com medidas tensionais abaixo destes níveis, mas com uso de medicação anti-hipertensiva. Entre as 1.020 pessoas entrevistadas, 267 (26,2\%; IC95\%: 23,5-28,9) apresentavam hipertensão arterial. Na regressão de Poisson encontrou-se associação estatisticamente significativa para idade, cor da pele, estado civil, história familiar, escolaridade e estado nutricional. As maiores frações atribuíveis foram entre as mulheres com até quatro anos de escolaridade (18,38\%); entre as obesas $(33,86 \%)$ e mulheres com sobrepeso (22,55\%). Os resultados foram comparados com outros estudos de prevalência realizados no estado e mostraram a importância da necessidade de articulação inter-setorial, revelando a contribuição possível de ser obtida mediante a elevação dos anos de escolaridade e salientaram a relevância das medidas de diminuição de peso na população.

Hipertensão; Estudos Transversais; Mulheres; Fatores de Risco

\section{Referências}

1. Hutchinson B, Birch S, Evans CE, Goldsmith LJ Markham BA, Frank J, et al. Selective opportunistic screening for hypercholesterolemia in primary care practice. J Clin Epidemiol 1998; 51:817-25.

2. Mansur AP, Favarato D, Souza MFM, Avakian SD, Aldrighi JM, César LAM, et al. Trends in death from circulatory diseases in Brazil between 1979 and 1996. Arq Bras Cardiol 2001; 76:504-10.

3. Núcleo de Informações em Saúde, Secretaria da Saúde do Rio Grande do Sul. Estatísticas de saúde: mortalidade 2002. Porto Alegre: Secretaria da Saúde do Rio Grande do Sul; 2003.

4. Olinto MTA, Dias-da-Costa JS, Mendes KG. A saúde em São Leopoldo. Relatório 2000. São Leopoldo: Programa de Pós-graduação em Ciências da Saúde, Universidade do Vale do Rio dos Sinos; 2003.

5. Chiavegatto Filho ADP, Laurenti R, Gotlieb SLD, Jorge MHPM. Mortalidade por doença hipertensiva em mulheres de 20 a 49 anos no Município de São Paulo, SP, Brasil. Rev Bras Epidemiol 2004; 7:252-8.

\section{Colaboradores}

O projeto de pesquisa foi elaborado por J. S. Dias-daCosta e M. T. A. Olinto, com a colaboração de Â. Tramontini. M. Hartmann e J. S. Dias-da-Costa participaram da análise e da redação do artigo. M. T. A. Olinto, M. P. Pattussi e Â. Tramontini participaram da análise e da revisão do texto.

\section{Agradecimentos}

Financiado pela Fundação de Amparo à Pesquisa do Estado do Rio Grande do Sul via Proad2 (n ${ }^{\circ}$. 02/0645,9) e Edital Universal 6 do Conselho Nacional de Desenvolvimento Científico e Tecnológico (n ${ }^{\circ}$. 473478/200-0), com apoio da Secretaria Municipal de Saúde de São Leopoldo. Parte de Dissertação de Mestrado apresentada ao Programa de Pós-graduação em Saúde Coletiva da Universidade do Vale do Rio dos Sinos, em 2006.
6. Lessa I. Epidemiologia da hipertensão arterial sistêmica e da insuficiência cardíaca no Brasil. Rev Bras Hipertens 2001; 8:383-92.

7. Barros FC, Victora CG, Vaughan JP. Epidemiologia da saúde infantil. Um manual para diagnósticos comunitários. São Paulo: Editora Hucitec/Fundo das Nações Unidas para a Infância; 1990.

8. Sociedade Brasileira de Hipertensão. III Consenso Brasileiro de Hipertensão Arterial. Campos do Jordão: Sociedade Brasileira de Hipertensão; 1998.

9. Chobanian AV, Bakris GL, Black HR, Cushman WC, Green LA, Izzo Jr. JL, et al. The seventh report of the Joint National Committee on Prevention, Detection, Evaluation, and Treatment of High Blood Pressure: the JNC 7 report. JAMA 2003; 289:2560-71.

10. Associação Nacional de Empresas de Pesquisa. Critério de classificação econômica Brasil. http:// www.anep.org.br/ (acessado em 23/Mai/2005). 
11. World Health Organization. Obesity: preventing and managing the global epidemic. Report of a WHO Consultation on Obesity. Geneva: World Health Organization; 1998.

12. Secretaria de Políticas de Saúde, Ministério da Saúde. Programa Nacional de Promoção da Atividade Física "Agita Brasil": atividade física e sua contribuição para a qualidade de vida. Rev Saúde Pública 2002; 36:254-6.

13. Moreira LB, Fuchs FD, Moraes RS, Bredemeier M, Cardoso S, Fuchs SC. Alcoholic beverage consumption and associated factors in Porto Alegre, a Southern Brazilian city: a population-based survey. J. Studies Alcohol 1996; 57:253-9.

14. Last JM. A dictionary of epidemiology. New York: Oxford University Press; 2001.

15. Barros AJ, Hirakata VN. Alternatives for logistic regression in cross-sectional studies: an empirical comparison of models that directly estimate the prevalence ratio. BMC Med Res Methodol 2003; 3:21.

16. Lessa I. O adulto brasileiro e as doenças da modernidade: epidemiologia das doenças crônicas nãotransmissíveis. 1a Ed. São Paulo: Editora Hucitec; 1998.

17. Achutti A, Medeiros AMB, Azambuja MIR, Costa EA, Klein CH. Hipertensão arterial no Rio Grande do Sul. Boletim da Saúde do Rio Grande do Sul 1985; 12:6-54.

18. Duncan BD, Schmidt MI, Polanczyk CA, Homrich CS, Rosa RS, Achutti AC. Fatores de risco para doenças não-transmissíveis em área metropolitana na região sul do Brasil. Prevalência e simultaneidade. Rev Saúde Pública 1993; 27:143-8.
19. Fuchs FD, Moreira LB, Moraes RS, Bredeneir M Cardozo SC. Prevalência de hipertensão arterial sistêmica e fatores associados na região urbana de Porto Alegre: estudo de base populacional. Arq Bras Cardiol 1995; 63:473-9.

20. Gus I, Harzheim E, Zaslavsky C, Medina C, Gus M. Prevalência, reconhecimento e controle da hipertensão arterial sistêmica no Estado do Rio Grande do Sul. Arq Bras Cardiol 2004; 83:424-8.

21. Piccini RX, Victora CG. Hipertensão arterial sistêmica no sul do Brasil: prevalência e fatores de risco. Rev Saúde Pública 1994; 28:261-7.

22. Trindade IS, Heineck G, Machado JR, Ayzemberg H, Formighieri M, Crestani M, et al. Prevalência de hipertensão arterial sistêmica na população urbana de Passo Fundo, RS. Arq Bras Cardiol 1998; 71:127-30.

23. Billings J, Zeitel L, Lukomnik J, Blank AE, Newman L. Impact of socioeconomic status on hospital use in New York City. Health Aff 1993; 12:162-73.

24. Sawyer DO, Leite IC, Alexandrino R. Perfis de utilização de serviços de saúde no Brasil. Ciênc Saúde Coletiva 2002; 71:757-76.

25. Culler SD, Parchman ML, Przbylsky M. Factors related to potentially preventable hospitalizations among elderly. Med Care 1998; 36:804-17.

26. Gus M, Fuchs SC, Moreira LB, Wiehe M, Silva AF, Albers F, et al. Association between different measurements of obesity and the incidence of hypertension. Am J Hypertens 2003; 17:50-3.

27. Jacoby E. The obesity epidemic in the Americas: making healthy choices the easiest choices. Rev Panam Salud Pública 2004; 15:278-84.

28. Rothman KJ, Greenland S. Modern epidemiology. Philadelphia: Lippincott Williams \& Wilkins; 1998.

Recebido em 13/Nov/2006

Versão final reapresentada em 23/Jan/2007 Aprovado em 06/Fev/2007 